\title{
Progress in Sub-Grid Scale Modeling of Shock-Turbulence Interaction
}

\author{
Alfred C. Buckingham \\ Jacob Grun
}

December 1994

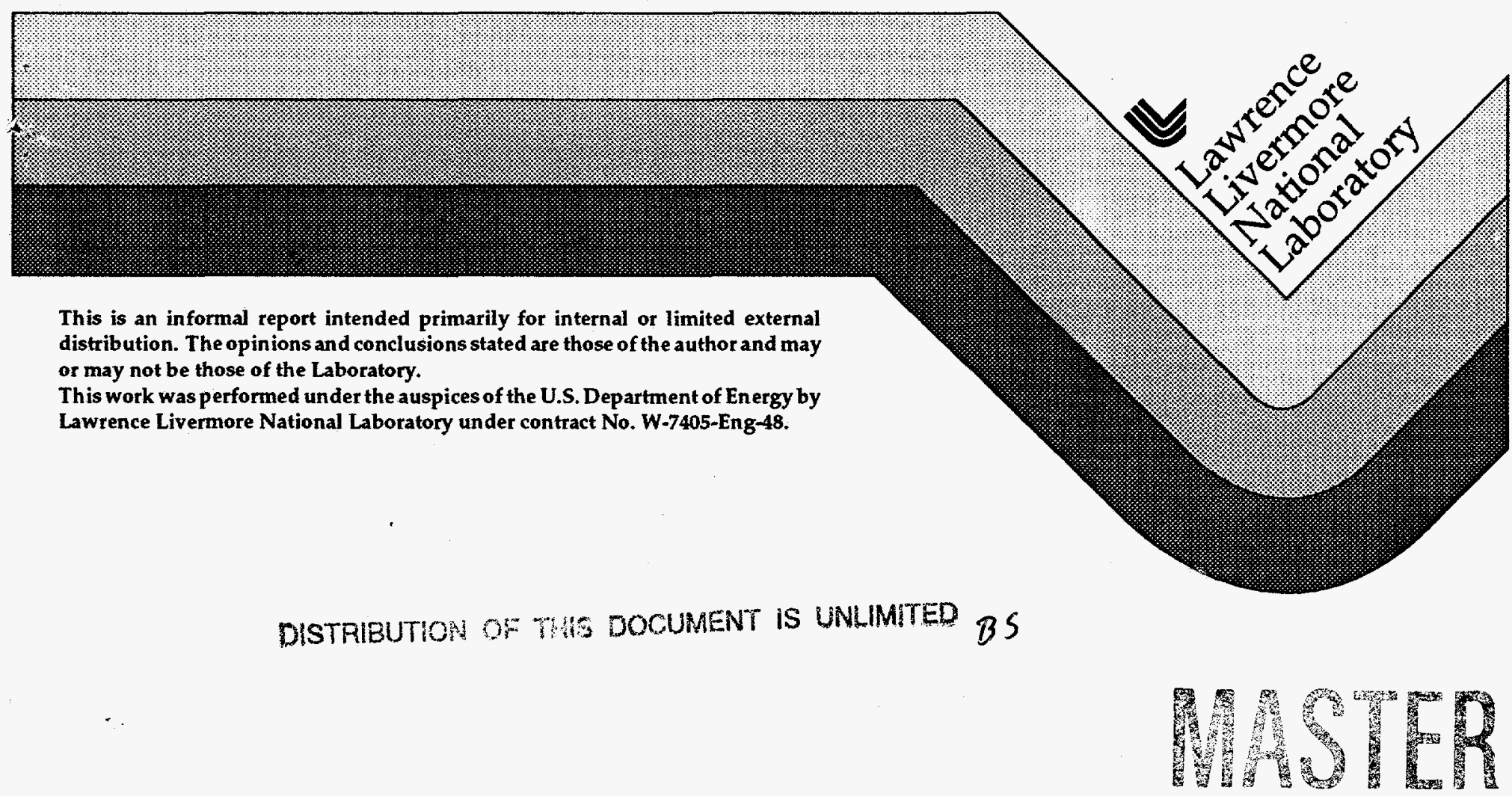




\section{DISCLAIMER}

Portions of this document may be illegible in electronic image products. Images are produced from the best available original document. 


\title{
/ EXTENDED ABSTRACT /
}

\section{PROGRESS IN SUB-GRID SCALE MODELING OF SHOCK-TURBULENCE INTERACTION}

\author{
Alfred C. Buckingham* and Jacob Grun** \\ *Center for Advanced Fluid Dynamics Applications (CAFDA) \\ H-Division, Physics and Space Technology Directorate \\ Lawrence Livermore National Laboratory, University of California \\ P.0. Box 808, Livermore, California 94551 \\ **Plasma Physics Division, Beam Physics Branch, Code 6795 \\ Naval Research Laboratory \\ 4555 Overlook Avenue SW \\ Washington DC 20375-5000
}

\begin{abstract}
PRECIS'
We report on progress in the development of sub grid scale (SGS) closure relationships for the unresolved motion scales in compressible large eddy simulations (LES). In our work, we emphasize flows evolving from an interactive juxtaposition of shock wave and turbulent field dynamics. In previous work we combined direct, twodimensional Monte Carlo shock structure simulations with compressible, explicit finitedifference Navier-Stokes approximations to the evolution of the resolved scale strain rate field. A modified Smagorinsky balance relationship was developed for modeling the unresolvable SGS motions. The balance production/dissipation components included buoyancy and shear (both bulk/dilatational and deformational) strain rate production and functionally parallel dissipation components. At present we are refining the SGS model and overall LES procedure to include: a linearized viscoelastic model for finite thickness shock distortions and shocked turbulence field response; multiple scale asymptotic considerations to improve predictions of average near-wall surface behavior, and a spectral statistical model simulating the effects of high wave number stochastic feed-back from the unresolved SGS nonlinear motion influences on the explicitly resolved grid scale motions. The explicit, fully-resolved strain field is computed using a conventional fractional time step, second order finite difference algorithm. The turbulence-distorted shockwave is captured as a discrete entity. Predicted amplification levels, modal energy partition, shock translational to turbulence kinetic energy transfer, and viscoelastic spatio-temporal response of turbulence to shock interaction are examined in comparison with available experimental evidence. Supplemental hypersonic compressible turbulence experimental information is developed from sub nanosecond interval pulsed shadowgraph evidence of laser impulse generated hypervelocity shocks interacting with intense, previously developed and carefully characterized initial turbulence. These data are obtained in the hypersonic shock Mach number range from 30 to more than 100 and in the background flow unit Reynolds number range from the order of $10^{5}$ to $10^{7}$. These simulations and experimental results are intended to provide the data base for test and refinement of more conventional turbulence models with the goal of providing computationally efficient, yet usefully and consistently accurate prediction of average turbulent transport and diffusion in the presence of almost ubiquitous, supersonic flow encountered, shockwave turbulence interactions.
\end{abstract}




\section{BACKGROUND}

This is a discussion of progress in continuing efforts to understand and predict the influence of shock waves on turbulent fields during shock-turbulence interactions. Observations show that the Reynolds stress tensor magnitude increases. Strong support for tensor amplification is supplied by the sharply distorted strain field localized to the immediate neighborhood of the shock surface. Beyond this there develops a gradual decay to a new more modestly amplified state relative to the pre-shocked level. Depending on the strength of the shock, the ambient pre-shocked conditions, and the intensity of the pre-shocked turbulence, the interaction may cause severe distortion, wave diffraction, or even complete decomposition of the shock front [Buckingham $(1989,1990)]$.

Practical interest is centered on the significantly altered, albeit shock localized, postshock turbulent kinetic energy, transport, component mixing, wall shear, and heat transfer. Fundamental questions remain on the nature of the amplification process and the energetic exchanges between translational shock energy, shock structure, and the turbulent field. Accurate description of the influence of shock-turbulence interactions is vital for predicting their influence on: supersonic/hypersonic flow field analysis, aerodynamic design, and aerostructural materials selection. Practical applications also include interior supersonic combustion analysis and combustion chamber design. It is also the essential foundation for accurately predicting the development and evolution of flow-field generated thermal and electromagnetic radiation important to hypersonic flight vehicle surviveability, detection and communication.

Combinations of low upstream turbulent intensity and strong shocks bring about the most pronounced changes in the level of turbulent intensity. Contrastingly, for weak shocks and intense upstream turbulence, shock distortions, and localized shock surface motions are most evident. Some of the observed consequences appear as a tendency for adiabatic weakening of the interactive shock Hugoniot jump conditions relative to values for a non interactive shock of the same strength and the same ambient pre-shocked conditions [Buckingham (1993), Buckingham and Grun (1993), Buckingham (1994a)].

It is a commonly held view that if the interactive shock Mach number is in the hypersonic range (shock Mach numbers equal to or greater than about 6), rapid reinforcement of shock strength at the front by non-linear pressure field interactions and consequent "self-healing" of shock front geometric irregularity act to suppress front distortion and also suppress the influence of the shock interactions on turbulence. However, very recent experimental evidence suggests that for intense upstream turbulence, significant shock distortions may develop in very strong, hypervelocity shock fields [Grun et al (1992), Grun et al (1993)]. Pronounced shock front distortion and substantial interactive modification of the turbulence seems to persist even for shocks propagating at shock Mach numbers well in excess of 100. For shock Mach Numbers less than six two dimensional modifications to classical Monte Carlo shock structure methods [ Bird (1967)] provided useful guidance on non uniform distortion and displacement of shocks interacting with turbulence in development of our earliest LES algorithms [Buckingham (1989), Buckingham (1990)]. At higher Mach Numbers, Monte Carlo collection and sampling requirements are computationally impractical in more than one dimension. A viscoelastic shock response model was developed using a kinetic theory approach for eddy shear and deformatioal response analagous to bulk and shear viscosity components in molecular gas transport [Buckingham (1992), Buckingham (1993), Buckingham (1994a)]. 
A primary goal of the present work is to provide a supplementary, high precision, numerical simulation tool for augmenting the sparse experimental evidence on shock turbulence interactions at these elevated Mach Numbers. Requisite modification of conventional compressible turbulence single point closure schemes may then be implemented and evaluated with the assistance of both simulation generated and experimentally generated and simulation analyzed data sets.

\section{REPRESENTATIVE DISCUSSION TO APPEAR}

Study of the details of the shock-turbulence interaction presents a daunting experimental and theoretical problem. For example, separation, isolation, and evaluation of the concomitant influences of entropy, velocity, and temperature fluctuations is forbiddingly difficult experimentally. This is particularly evident in the presence of strong shocks. Here the classical analytical procedures for linearized isolation and superposition of these influences is invalid and usually produces unfortunately misleading experimental interpretations.

Theoretical analysis, assisted by computer model studies and direct Navier-Stokes numerical simulations have provided some assistance and guidance in the design of experiments, refinement of diagnostics and in experimental interpretation. However, even with enhanced resolution obtained by use of adaptive grid techniques, multigrid procedures and/or spectral methods (where the shock could be isolated by analytical shock fitting schemes), the results are usually severely under resolved for all but the lowest Reynolds Number scale flow situations [Buckingham (1989), Buckingham (1990)].

LES procedures which explicitly follow the largest scale motions while carefully partitioning and incorporating the ensemble averaged influence of the non-resolvable scales appear to be an intermediate (and perhaps long term) answer to this dilemma.

This paper will illustrate our progress in developing a successful LES technique with particular attention to computational applications where the presence and interaction of strong shocks with compressible turbulent fields is of paramount importance. Our specific focus at this stage is on the sub-grid problem. As concisely stated in a recent LES review and application [Leith (1990)] in sub-grid scale modeling considerations, the turbulence problem is reduced to treating, as well as one can, the effect of the unresolved subgrid scales on those which are explicitly computed. This also tends to place the SGS modeling problem in proper perspective with respect to the encompassing LES development.

Our current efforts emphasize more subtle but significant influence of fluctuations on shock geometry and surface boundary conditions [Emanuel and Liu (1988), Lele (1992)]; conditional constraints based on multi-time scale asymptotic considerations [Speziale et al (1988)]; informative parallels between the interactive shock structure viscoelastic response model relationships and analagous kinetic theory moment developments of first and second molecular viscosity relationships in shock structure theory [Buckingham (1992), Buckingham (1994a \& 1994b)].

As demonstrated in recent compressible large eddy simulation developments [Erlebacher (1990), Leith (1993)] the unresolvable sub-grid scales have two distinct influences on the resolvable grid scale motions. One is the well known influence on dissipation of the energy in the computable scales. The other, lesser recognized is the spectral range stochastic influence of non-linear interactions between the small scales which feed back into the large scale motions. 
While less recognized, this other influence has been well established for two decades or more. Stochastic models of turbulence were introduced by Kraichnan about 30 years ago [Kraichnan (1967)] in order to guarantee the realizability of the derived statistical moments. These models replace the original quadratically nonlinear chaotic dynamics by a random linear model of the Langevin sort with damping and random forcing terms determined by the statistical properties of the system. As is well known such models can be used to develop subgrid scale models for large eddy simulation (LES) which include not only a wavenumber $\mathrm{K}$ dependent eddy viscosity but also a stochastic forcing of resolved scales with a variance spectrum proportional to $k^{4}$ (strictly suitable for homogeneous, isotropic fields). In studies of the predictability of turbulent flows, it is such random forcing that contaminates the deterministic nature of the large scale motion and exhibits the chaotic nature of turbulence.

The widely used K- $\varepsilon$ model for inhomogeneous engineering turbulence suffers from treating all random scales as modeled turbulence. This works well when the inhomogeneity and properties of interest are essentially one-dimensional, but in the numerical simulation of two- and three-dimensional flows it is desirable to recapture explicitly those eddy structures large enough to be resolved, which is the principal aim of LES. For several decades the eddy damping aspect of subgrid scale modeling has been treated with a local nonlinear version of the Smagorinsky eddy viscosity prescription [Smagorinsky (1963)]. More recently this viscosity has been supplemented with stochastic backscatter in order to provide the appropriate forcing of resolved scales by the nonlinear coupling to subgrid scales of unknown phase [Leith (1990), Leith (1993), Buckingham (1993)].

In other work, we further develop and apply a linear viscoelastic response model at both the interaction deformed shock front as well as in the shocked turbulent field. The viscoelastic response of turbulence to sudden impulse has been identified and theoretically studied for several decades. For example, in [Kraichnan (1976)] an identification is made of other work on this phenomena from the previous decade including application of the Direct Interaction Approximation model to simulate and characterize viscoelastic response of an incompressible turbulent field to an imposition of stress.

The dynamic viscoelastic response model for the neighborhood of the shock front with exponential relaxation behind it is coupled to the shock free Smagorinsky subgrid model directly originally using relaxation and modulus parameters originally developed in the previously discussed Monte-Carlo shock structure simulations [Buckingham (1989), Buckingham (1990)] and later extended to a continuum description of the shock interaction region and the shocked turbulence region produced by it [Buckingham (1992), Buckingham (1993), Buckingham (1994a) \& (1994b)].

Briefly the LES procedural solution is approximated with discretized, time explicit, two-dimensional equations of compressible viscous flow, where averaging is massweighted (Favre, 1983) over the Smagorinsky modeled subgrid scale motions represented by a total, shear, buoyancy (bulk) and dilatarion contribution to the eddy viscosity, $v_{\mathrm{t}}$.

The viscoelastic eddy field response property is developed as in direct analogy with the rheological non-Newtonian transient state of the stress modulus, $v_{t}$, which may either decrease (thixotropic) or increase (rheopectric) with applied shear stress, $T_{i k}$, and then partially returns (relaxes) to a former state. 
In our work a simple, linear viscoelastic form is assumed.

$$
v_{t}=v_{t} \Phi+I v_{t} \pi\left(M, l_{u}\right)-v_{t} \Phi \operatorname{Iexp}-(\tau / \tau *)
$$

Here the metastable state, $v_{t} \pi$, is determined to be a function of both shock Mach No, $\mathbf{M}$, and relative intensity of the fluctuating velocity in the resolved scales, $\mathfrak{l}_{\mathrm{u}}$, while $\tau$ is the real time of application and $\tau *$ is the transient relaxation parameter. Both $v_{\mathrm{t}} \pi$ and $\tau *$ are developed from independent, stochastic shock turbulence interaction simulations, from experiment or from successive LES numerical relaxation trials. This part of the analysis is by no means complete and represents an important component of our on-going efforts in developing a consistently effective SGS model for shockwave turbulence interaction. The complexity of the development increases with dimensionality (2-dimensional and 3dimensional distinctions on the evolution of turbulent structure functions) and the possible appearance of shock caustics and shocklets in the evolving, shocked turbulent field following interaction [Lee, Lele, and Moin (1991)].

The third major component of our SGS developmental progress is associated with a description of the rapid variation in the dominant scales of motion (order of magnitude reduction in the immediate neighborhood of wall surfaces) and the corresponding influence on predicting the spectrally averaged transport behavior in order to provide adequate guidance for modifications of single point closure models for global (boundary layer and free shear layer) applications. The near wall SGS model behavior development is systematic with appeal to information from application of asymptotic multiple scales analysis [Buckingham (1992), Buckingham (1994a \& 1994b)].

Special guidance in developing more generally effective compressible subgrid scale models is obtained from some revealing combinations of theoretical 2 nd order moment closure analysis, first order asymptotic considerations and direct numerical simulations [Speziale et al (1988)] and from attendant subsequent analysis of the compressible subgrid scale closure process [Erlebacher et al (1990)]. Here advantage is taken of the appropriate use of direct numerical simulations to illustrate some crucial features of the wave dominated turbulent transport region in comparison to the rotationally dominated region.

In the Mach Number range below about 5 , there is an especially rich experimental data base for shockwave turbulent boundary layer interactions. We have selected one of the particularly complete sets of measurements from a set of 1987 experiments [Smits and Muck (1987)]. for most of our development, test and calibration of the near-wall shock turbulence simulations. This set of experiments was selected because of the unusual completeness of surface, near wall distributions of Reynolds stress and some elemental turbulent structure functions and for the availability of streamwise measurements at a significant number of stations upstream and downstream of the shockwave interaction for three distinct oblique shockwave turbulent boundary layer compression corner interaction situations.

Fig. 1 shows an ultra short time (sub-nanosecond) exposure shadowgraph. This is an instantaneous realization of a hypersonic spherical shock front moving (from bottom to top in the photo) through and interacting with a turbulent field. Digital enhancement has been used to reveal subtle interaction dynamics details for analysis. The ambient turbulent field, as yet unaffected by the shock, appears on the upper right part of the photo. 
The shocked turbulence with obviously contracted and distorted scales of motion appears below and behind the upward moving shock front in the photo. The substantial distortion of the shock front at the shock turbulence interface is also clearly apparent. Numerical large-eddy simulations are developed in a careful match of the instantaneous experimental flow conditions and their time and space evolution. The experimental and numerically simulated results form an expanded data base for detailed analysis.

Fig. 2 illustrates the shockwave amplification of the turbulence energy in terms of the power spectral density distribution before and after shock transit at a Mach number of about 100. The dashed curve shows the results of our current LES simulations in the incident turbulent field ahead of the advancing shock, while the results of the experimental observations appear as open circles. Immdiately after shock wave passage measured, solid circle, and computed solid curve illustrate the substantial energy amplification and implied transfer from directed shock energy to turbulence kinetic energy. The Kolmogorov inertial range decay $\left(\kappa^{5 / 3}\right)$ is provided as a phantom line for reference.

Fig. 3 summarizes some of the results in terms of the amplification of spatially averaged turbulent energy content as a function of distance behind the shock and the sudden reduction in size of the averaged scales of motion after shock transit. The information is used in the present investigation to help form a theoretical description of the transfer of directed shock wave energy into turbulence and the partition of energy over scales of random motion in the disturbed region behind the shock.

Fig. 4 summarizes the current results on the shock amplification of overall turbulence kinetic energy with respect to the available previous data base in terms of the shocked Mach number. Linearized theory based on superimposed perturbations to the shock jump conditions on a plane undistorted shock front predicts decay to negligible amplification at Mach numbers greater than about ten. This is shown as the solid curve on the graph. The previous experimental data is primarily shockwave turbulent boundary layer information with some free shock amplification experiments in the range of Mach numbers up to about 2.5. The experiments appear as solid circles. Our LES computational results appear as solid squares. The recent experimental results are symbolized by the open segmented squares at the higher (10 to 100$)$ Mach Number range. While only a single LES calculation (Mach 100) appears, others are being developed at present to help cover the range depicted by the recent experiments. The important message is that shock amplification of turbulence energy appears to be an important phenomena even in the hypersonic Mach number range.

It is suggested that the shockwave turbulence interaction problem is a prototypical physical process that is imperfectly understood and yet dominates many typical supersonic and hypersonic flow investigations. It is also a daunting numerical problem combining the special problems of resolving shockwaves and turbulence. This discussion will emphasize current progress in developing all-Reynolds-number, compressible LES procedures to assist us in our investigation of the underlying physical mechanisms that govern these interactions, and to provide substantive information for effective modification of computationally efficient single point closure schemes capable of consistently predicting these results with practical accuracy. 


\section{REFERENCES}

- Bird, G. A., (1967), J. Fluid Mech. 30, 479.

- Buckingham, Alfred C., (1989) "Numerical Studies of Shock Wave Structure Sensitivity to Interactions with Turbulent Fields, Numerical Methods in Laminar and Turbulent Flow, Vol. VI Part 1, Eds., C. Taylor, P.Gresho, R. L. Sani, J. Hauser, Pineridge Press, Swansea, UK, 805.

- Buckingham, A. C., (1990) "Interactive Shock Structure Response to Passage through Turbulence", AIAA Paper 90-1642, AIAA 21st Fluid and Plasma Dynamics Conf., Seattle, WA, 18-20 June, 1990.

- Buckingham, Alfred C. (1992) "Viscoelastic Influences in Compressible Large Eddy Simulations", Proc. Third International Workshop on the Physics of Compressible Turbulent Mixing, eds. B. Sitt, J-F Haas, N. Wilke, CEA, Centre d'Etudes de LimeilValenton and Centre d'Etudes de Vajours-Moronvillier, Paris.

- Buckingham, Alfred C. (1993) "Large Eddy Simulation of Shockwave Passage through Turbulence", Proc. Symposium on Engineering Applications of Large-Eddy Simulations, ASME Fluids Engineering Summer Meeting, (June 20-23, Washington DC.)

- Buckingham, Alfred C. and Grun, Jacob (1993) "Sub-grid Scale Modeling for LargeEddy Simulations in Analysis of Shock-Turbulence Interactions", Numerical Methods in Laminar and Turbulent Flow, Vol. VIII, Part 2. C. Taylor, ed. Pineridge Press, Swansea, UK, 1033.

- Buckingham, A. C. (1994a), "Shock Turbulence Interactions: I. The Shock Wave", Univ. Calif. LLNL, UCRL-JC-118434, Pt. 1, presented at Theoretical Fluids Group T3 Seminar, LANL, Los Alamos, NM, Oct, 1994.

- Buckingham, A. C. (1994b), "Shock Turbulence Interactions: II. The Turbulence", Univ. Calif. LLNL, UCRL-JC-118434, Pt. 2, presented at Theoretical Fluids Group T3 Seminar, LANL, Los Alamos, NM, Oct 1994.

- Emanuel, G., M.-S. Liu (1988), Phys. Fluids, 31, 3625.

- Erlebacher, G., M.Y. Hussaini, C. G. Speziaie, and T. A. Zang, (1990),"On the Large Eddy Simulation of Compressible Isotropic Turbulence", 12th International Conference on Numerical Methods in Fluid Dynamics, (University of Oxford, Oxford, UK, 9-13 July, 1990).

- Grun, J., Manka, C. K., Ripin, B. H., Buckingham, A. C., Resnick, J., and Burris, H. R. (1992) "Enhancement of Turbulence by a High Mach Number Shock", Bull.American Physical Sociery, Vol. 37, No. 6, p. 1478.

- Grun, J., Manka, C. K., Ripin, B. H., Ting, A., Buckingham, A. C., and Burris, H. R. (1993), "Interaction of Turbulence with Ultra-High Mach Number Shocks, Bull. American Physical Society, Vol 38, No. 6, (full paper in preparation).

- Kraichnan, R. H., (1967), Phys. Fluids 10, 1417. 
- Kraichnan, R. H. (1976)J. Atmos. Sci., 33, 1521.

- Lee, Sangsan, Sanjiva K. Lele, and Parviz Moin, (1991) "Eddy shocklets in decaying compressible turbulence" Phys. Fluids A 3 (4), 657.

- Leith, C. E., (1990) "Stochastic Backscatter in a Subgrid-scale Model: Plane Shear Mixing Layer", Phys. Fluids, A Vol. 2, 297.

- Leith, C. E., (1993) "Stochastic Backscatter in a Subgrid-Scale Model: 3D Compressible Flows", International Workshop on Large Eddy Simulation, eds. S. A. Orszag, T. A. Zang, Cambridge University Press, Cambridge, UK.

- Lele, Sanjiva K., (1992) "Shock-jump relations in a turbulent flow", Phys. Fluids A 4 (12), 2900.

- Smagorinsky, J., (1963) Mon. Weather Rev. Vol. 91, 99.

- Smits, Alexander J. and Muck, Kin-Choong, (1987) "Experimental Study of Three Shock Wave/Turbulent Boundary Layer Interactions", J. Fluid Mech. Vol. 182, 291.

- Speziale, C. G., G. Erlebacher, T. A. Zang, and M. Y. Hussaini, (1988), "The subgrid scale modeling of compressible turbulence", Phys. Fluids 31, 940.

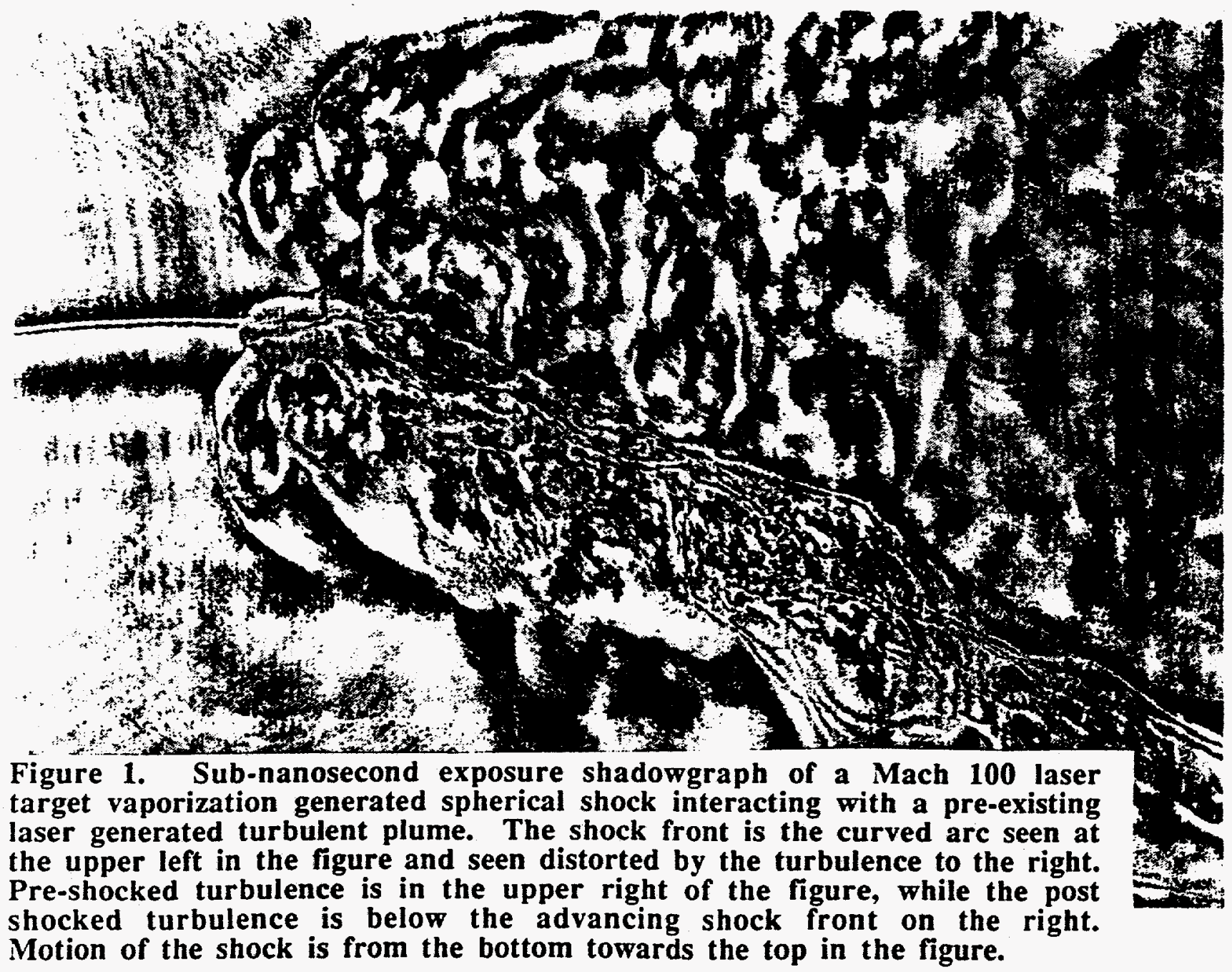




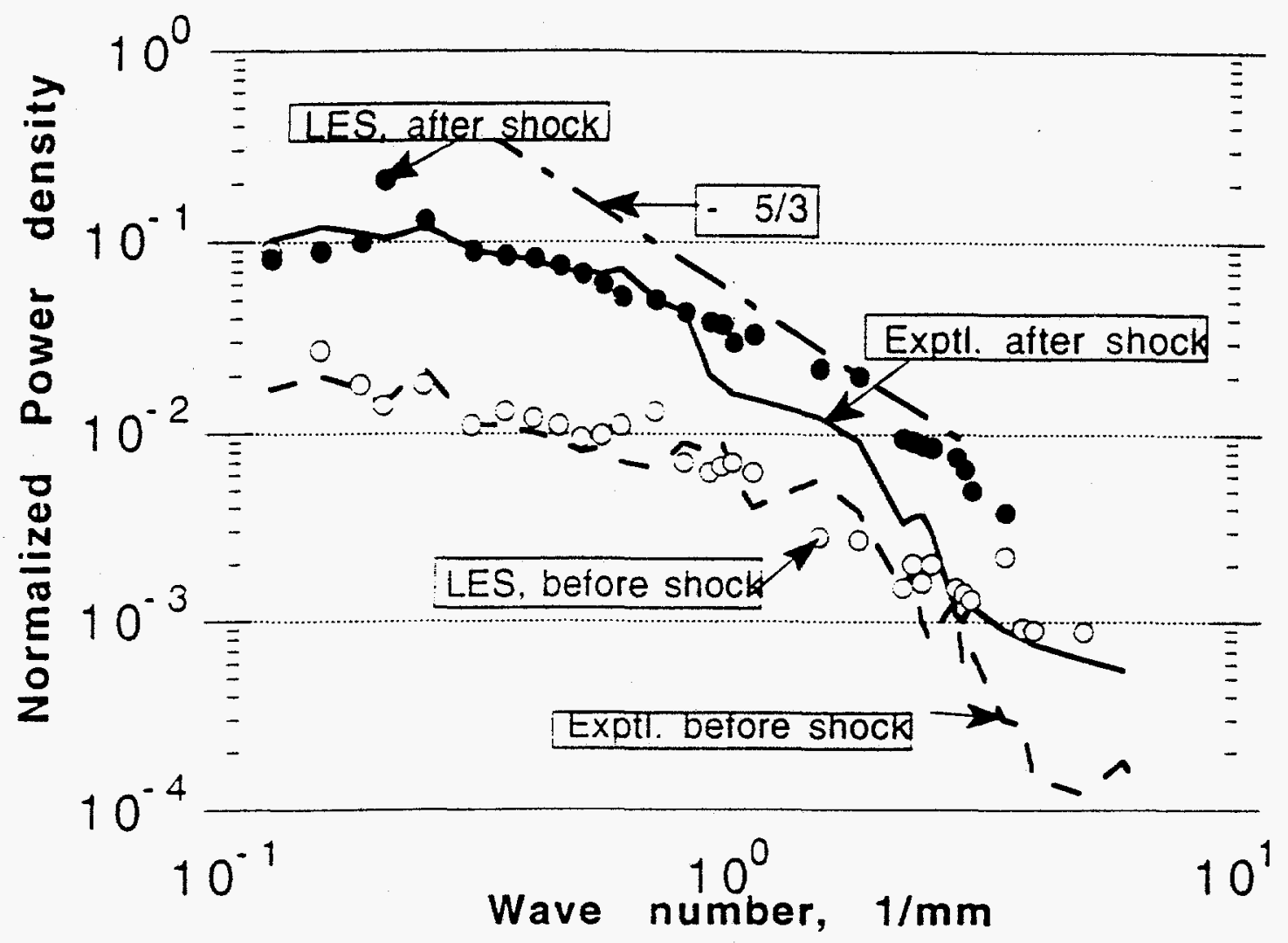

Figure 2. Normalized Power Density spectra before and after shock interaction at a shock Mach Number of 100. Experimental data from Grun et al (1992 \& 1993). LES results from current calculations using the techniques described in this paper. 


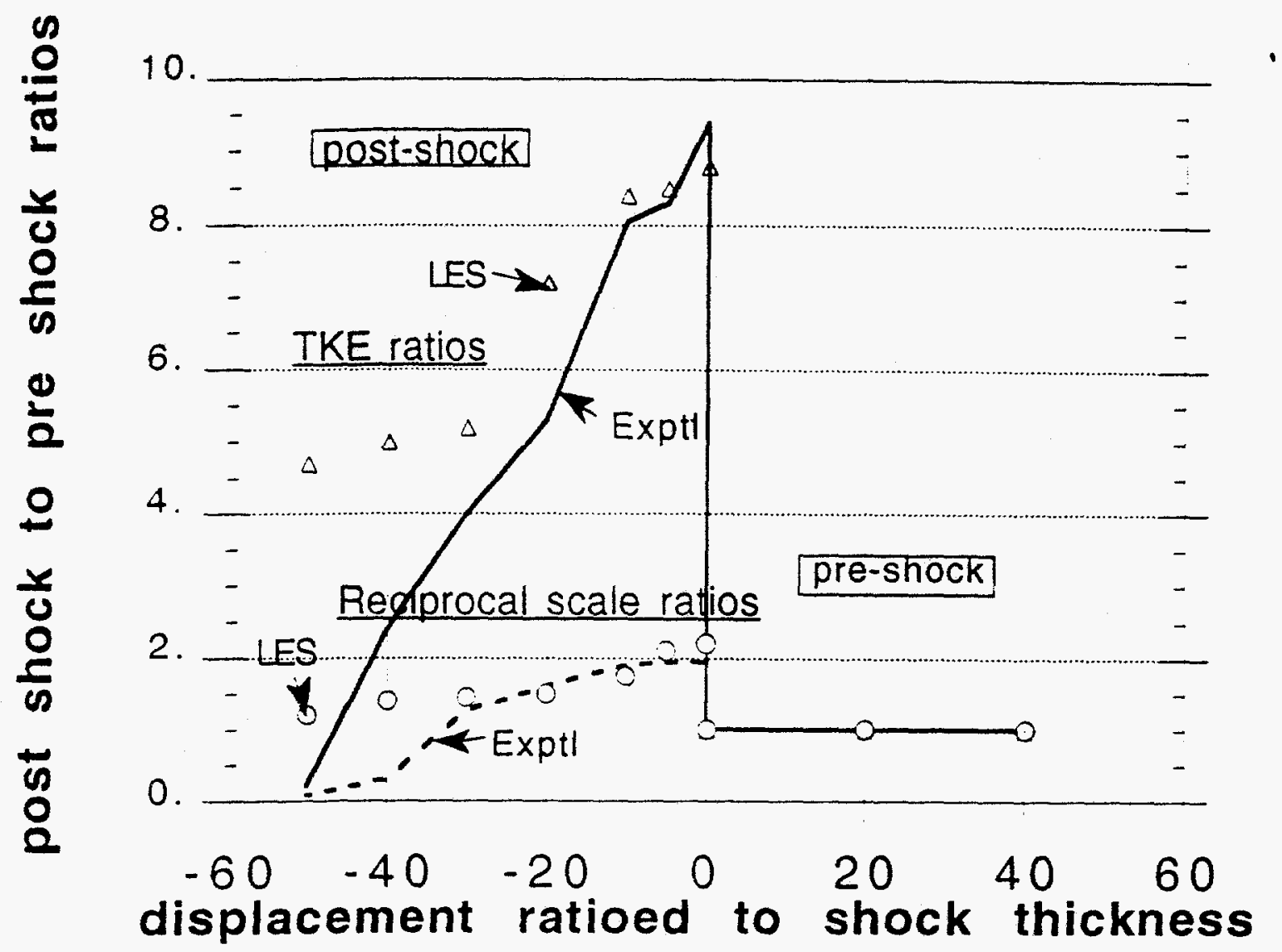

Figure 3. Comparison of turbulent kinetic energy amplification and statistical correlation length scale contraction through and beyond shock interaction as measured in the current laser generated spherical shock turbulence interaction experiments and simulated in detail using the compressible LES procedure described in this investigation. 


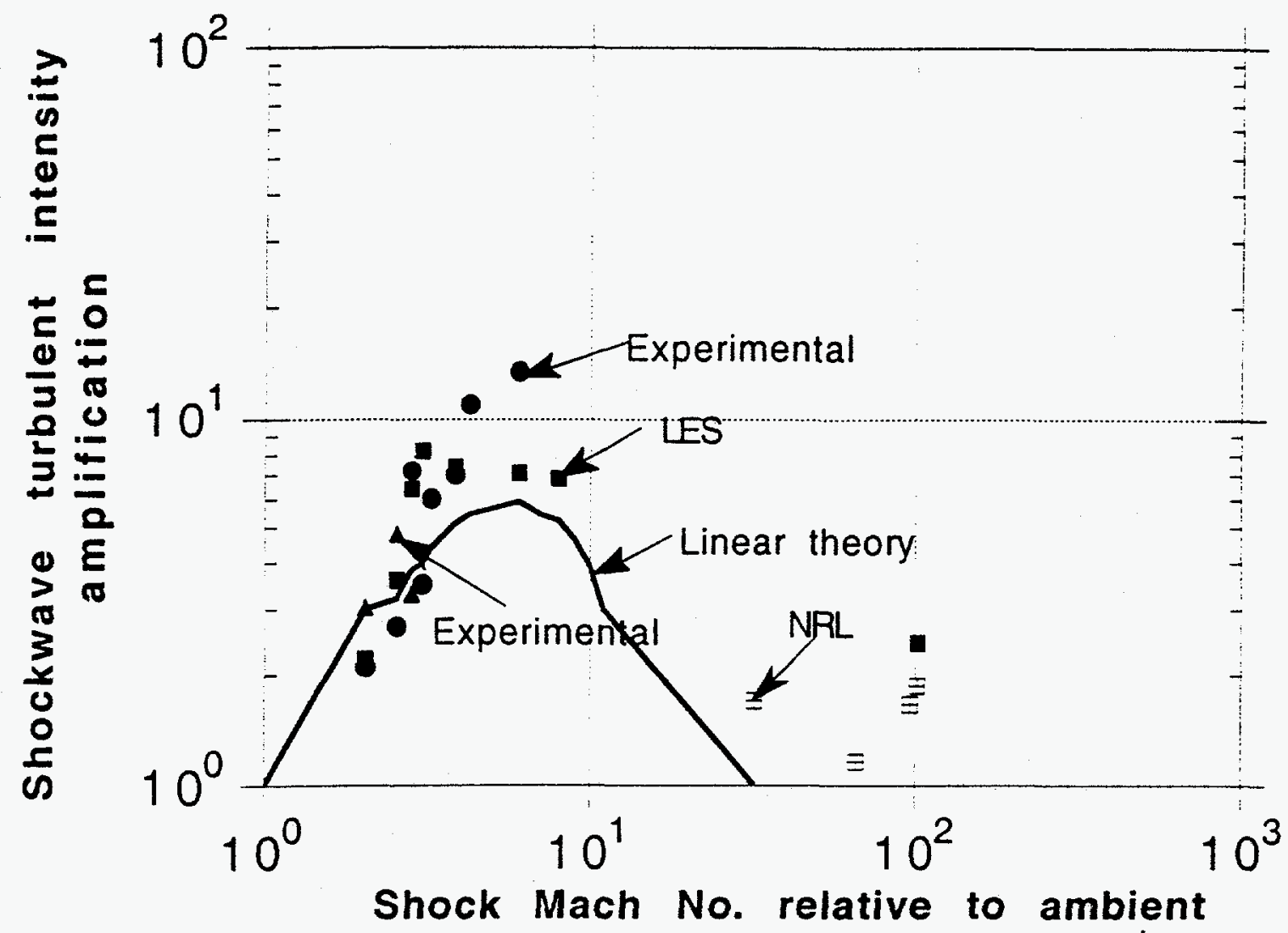

Figure 4. Experimental and computed results on shock amplification of initial (unchocked) turbulence kinetic energy for a Mach number range of 1 to 100 . The computed results were produced by current and earlier versions of the LES techniques described in this paper. 